\title{
Cluster Synchronization of Stochastic Complex Networks with Markovian Switching and Time-Varying Delay via Impulsive Pinning Control
}

\author{
Xuan Zhou and Kui Luo \\ Industrial Training Center, Shenzhen Polytechnic, Shenzhen 518000, China \\ Correspondence should be addressed to Kui Luo; luokui@szpt.edu.cn
}

Received 4 April 2014; Revised 4 June 2014; Accepted 4 June 2014; Published 26 June 2014

Academic Editor: Luca Guerrini

Copyright @ $2014 \mathrm{X}$. Zhou and K. Luo. This is an open access article distributed under the Creative Commons Attribution License, which permits unrestricted use, distribution, and reproduction in any medium, provided the original work is properly cited.

This paper studies the cluster synchronization of a kind of complex networks by means of impulsive pinning control scheme. These networks are subject to stochastic noise perturbations and Markovian switching, as well as internal and outer time-varying delays. Using the Lyapunov-Krasovskii functional, Itö's formula, and some linear matrix inequalities (LMI), several novel sufficient conditions are obtained to guarantee the desired cluster synchronization. At the end of this writing, a numerical simulation is given to demonstrate the effectiveness of those theoretical results.

\section{Introduction and Model Description}

Complex networks can be used to describe properly many biological, social, and communication systems and are made up of a great number of nodes representing individuals or organizations and links that are employed to mimic the interactions among them [1]. In particular, interesting examples are the spatiotemporal chaos [2], the Internet $[3,4]$, spiral waves [5], and the World Wide Web [6]. Synchronization is a universal phenomenon in nature, has potential applications significantly in real-world dynamical systems, and can be understood as the adjustment of coherence or rhythms of all states through interaction $[7,8]$. Hitherto, a lot of different synchronization patterns have been investigated such as complete synchronization [9], phase synchronization [10], partial synchronization [11], and cluster synchronization [12]. Actually, the synchronization patterns can be nearly contained by a uniform definition.

In brief, the cluster synchronization can be understood as the dynamical nodes synchronize each other in each group, while there is no synchronization between any two different groups. A large number of real-world networks exhibit community structure, for example, communication engineering and biological science [13]. Cluster synchronization in twodimensional and three-dimensional lattices of diffusively coupled chaotic oscillators was discussed in [14]. Cluster synchronization in a kind of strictly semipassive complex networks by means of diffusive coupling was focused on in [15]. In [16], Ma et al., under the help of a special coupling matrix for some connected chaotic networks, expressed that the randomly selected cluster synchronization manifolds could be stabilized. A novel and effective approach was provided to reach cluster synchronization of complex network and ensure their stability for a given nearest neighborhood network with zero-flux or periodic boundary conditions. In a few words, cluster synchronization, due to its significant practical implications, was considered widely and deeply by many researchers from various fields.

Moreover, owning to uncertain interferences caused by man-made and particularly natural factors, perturbation is a very universal and vital property in many real systems. There are lots of previous works contributing to studying stochastic phenomena of complex networks. For instance, in [17], the authors considered the case where only a single node was affected by stochastic perturbations. Reference [18] talked over an array of networks with scalar Wiener processes, which implied that each node is influenced by the same noise. Apparently, this assumption might be a little unrealistic for the real-world networks which were commonly influenced by different multidimensional perturbations. In [19], the writers 
discussed a class of coupled neural networks with stochastic noise and intermittent control. Also, their Wiener processes were of a vector form that can be viewed as an improvement of [18]. Consequently, the complex networks should be and must be brought in stochastic perturbations.

Recently, more and more researchers turn their attention to switched system in control theory domain. Generally speaking, a switched system includes two basic elements at least: one is several dynamical subsystems and the other one is a switching law used to specify some active subsystem at each instant of time. In view of different characteristics of complex networks, switching law also has several distinct patterns. For example, [20] probed into a blinking network whose switching couplings were according to certain probability. The writing pointed out that this kind of networks could synchronize for nearly all instances of the fast random switching process. As a matter of fact, from the mathematical point of view, switching signals between different network models can be governed by a Markovian chain. Recurring to linear matrix inequality, [21] considered the synchronization of a class of complex networks with Markovian jumping parameters whose coupling configuration was not dependent on mode switching. In addition, the synchronization issue in an array of neural networks with mixed delays and stochastic hybrid jumping couplings was investigated in [22] by adaptive control scheme. Nevertheless, if all nodes placed restriction on sharing common time-delay in a Markovian chain, this will lead to unpractical results. However, as far as I am concerned, the result on synchronization of stochastic networks with Markovian switching and time-varying delay coupling is seldom.

In view of the preceding discussion, this paper will focus on the issue of the synchronization of stochastic complex networks with Markovian switching and time-varying delay coupling by impulsive pinning control method. By the aid of the Lyapunov-Krasovskii functional method and some common linear matrix inequality in this field, several valid sufficient conditions will be obtained to ensure the cluster synchronization.

This paper is organized as follows. In Section 2, we introduce the generally considered model for a stochastic complex network. Moreover, some preliminary definitions and theorems needed for the rest of the paper are also provided. Section 3 shows some synchronization criteria for the discussed complex networks by logic and pleasing proof. In Section 4, three numerical simulations are given to illustrate our theoretical results. Ultimately, the paper concludes in Section 5.

Notations. In this paper, the superscript $T$ will denote the transpose of a matrix or a vector. $\mathbb{R}^{n}$ will denote the $n$ dimensional Euclidean space and $\mathbb{R}^{n \times n}$ the set of all $n \times n$ real matrices. $\mathbf{1}_{n}=(1,1, \ldots, 1)^{T} \in \mathbb{R}^{n}$, and $I_{n}$ is an $n$ dimensional identity matrix. $\operatorname{Tr}(\cdot)$ stands for the trace of the corresponding matrix. For square matrix $M$, the notation $M>0(<0)$ will mean that $M$ is a positive-definite (negativedefinite) matrix. $\lambda_{\max }(A)$ and $\lambda_{\min }(A)$ will denote the greatest and least eigenvalues of a symmetric matrix, respectively, and $\check{p}=\max \left\{p_{1}, p_{2}, \ldots, p_{n}\right\}, \widehat{p}=\min \left\{p_{1}, p_{2}, \ldots, p_{n}\right\}$.

\section{Preliminaries}

Let $\left(\Omega, \mathscr{F},\left\{\mathscr{F}_{t}\right\}_{t \geq 0}, \mathscr{P}\right)$ be a complete probability space with a filtration $\left\{\mathscr{F}_{t}\right\}_{t \geq 0}$ that is right continuous with $\mathscr{F}_{0}$ including all the $\mathscr{P}$-null sets. $C\left([-\tau, 0] ; \mathbb{R}^{n}\right)$ will denote the family of continuous functions $\phi$ from $[-\tau, 0]$ to $\mathbb{R}^{n}$ with the uniform norm $\|\phi\|^{2}=\sup _{-\tau \leq s \leq 0} \phi(s)^{T} \phi(s)$. And $C_{\mathscr{F}_{0}}^{2}\left([-\tau, 0] ; \mathbb{R}^{n}\right)$ denote the family of all $\mathscr{F}_{0}$ measurable, $C\left([-\tau, 0] ; \mathbb{R}^{n}\right)$-valued stochastic variables $\xi=\{\xi(t):-\tau \leq t \leq 0\}$ such that $\int_{-\tau}^{0} \mathbb{E}|\xi(t)|^{2} d t \leq \infty$, where $\mathbb{E}$ stands for the correspondent expectation operator with respect to the given probability measure $\mathscr{P}$.

As follows, some definitions, lemmas, and notations are revealed which will be used throughout this paper.

Definition 1. If $A=\left[a_{i j}\right] \in R^{m \times m}$ is an irreducible matrix such that $a_{i j}=a_{j i} \geq 0$ for all $i \neq j$ and $\sum_{j=1}^{m} a_{i j}=0$ for all $i=1,2, \ldots, m$, one says that $A \in \mathbf{A}_{\mathbf{1}}$.

Definition 2 (see $[16,23])$. Let

$$
A=\left[\begin{array}{cccc}
A_{11} & A_{12} & \cdots & A_{1 d} \\
A_{21} & A_{22} & \cdots & A_{2 d} \\
\vdots & \vdots & \ddots & \vdots \\
A_{d 1} & A_{d 2} & \cdots & A_{d d}
\end{array}\right]
$$

be an $m \times m$ symmetric matrix such that $A_{u v} \in R^{k_{u} \times k_{v}}, u, v=$ $1,2, \ldots, d$. If each block $A_{u v}$ is a zero-row-sum matrix, one says that $A \in \mathbf{M}_{1}$. Furthermore, if $A_{u u} \in \mathbf{A}_{1}, u=1,2, \ldots, d$, one says that $A \in \mathbf{M}_{2}$. It also follows from the symmetry of $A$ that $A_{u v}=A_{v u}^{T}, A_{u v}, A_{v u}$ are zero-row-sum matrices and $A_{u v}$ is a zero-column-sum matrix.

Remark 3. Definition 2 defines a diffusively coupled matrix; if $a_{i j}>0$ for $i \neq j$, then the coupling between $i$ and $j$ is called cooperative coupling; if $a_{i j}<0$ for $i \neq j$, then the coupling between $i$ and $j$ is called competitive coupling [16].

Before discussing the synchronization of the considered complex networks, we will introduce the mathematical definition of cluster synchronization.

Definition 4 (see [24]). Let $G_{1}=\left\{1,2, \ldots, k_{1}\right\}, G_{2}=\left\{k_{1}+\right.$ $\left.1, k_{1}+2, \ldots, k_{1}+k_{2}\right\}, \ldots, G_{d}=\left\{k_{1}+k_{2}+\cdots+k_{d-1}+1, \ldots, k_{1}+\right.$ $\left.k_{2}+\cdots+k_{d}\right\}$ be a partition of the set $G=\{1,2, \ldots, m\}$ for $1<d<m, 1<k_{l}<m$, and $\sum_{l=1}^{d} k_{l}=m$. Moreover for every $i \in G$, let $\check{i}$ be the counting index of the subset in which the number is $i$; that is, $i \in G_{\breve{i}}$. A network with $m$ nodes is said to realize cluster synchronization with partition $\left\{G_{1}, G_{2}, \ldots, G_{d}\right\}$, if the state variables of the nodes satisfy $\lim _{t \rightarrow+\infty}\left\|x_{i}(t)-x_{j}(t)\right\|=0$ for $\check{i}=\check{j}$ and $\lim _{t \rightarrow+\infty} \| x_{i}(t)-$ $x_{j}(t) \| \neq 0$ for $\check{i} \neq \check{j}$ for all initial values. 
The state equation of the switched network consisting of $N$ identical nodes without delay and time-varying delay coupling and Markovian jumping are given as follows:

$$
\begin{aligned}
& d x_{i}(t)=\left\{f_{\breve{i}}\left(t, x_{i}(t), x_{i}(t-\tau(t))\right)\right. \\
&+\sum_{j=1, i \neq j}^{N} a_{i j}^{(r(t))} \Sigma\left(x_{j}(t)-x_{i}(t)\right) \\
&+\sum_{j=1, i \neq j}^{N} b_{i j}^{(r(t))} \sum\left(x_{j}\left(t-\tau_{c}(t)\right)\right. \\
&\left.\left.\quad-x_{i}\left(t-\tau_{c}(t)\right)\right)\right\} d t \\
&+\sigma_{i}\left(t, x(t), x(t-\tau(t)), x\left(t-\tau_{c}(t)\right), r(t)\right) \\
& \times d w_{i}(t), \quad i=1,2, \ldots, N,
\end{aligned}
$$

where $x_{i}(t)=\left(x_{i 1}(t), x_{i 2}(t), \ldots, x_{i n}(t)\right)^{T} \in \mathbb{R}^{n}$ is the state vector of the $i$ th node of the network, $f\left(t, x_{i}(t), x_{i}(t-\right.$ $\tau(t)))=\left[f_{1}\left(t, x_{i}(t), x_{i}(t-\tau(t))\right), f_{2}\left(t, x_{i}(t), x_{i}(t-\tau(t))\right), \ldots\right.$, $\left.f_{n}\left(t, x_{i}(t), x_{i}(t-\tau(t))\right)\right]^{T}$ is a continuous vector-valued function, and $r(t)$ are the continuous-time Markov processes that describe the evolution of the modes at time $t . \Sigma=$ $\operatorname{diag}\left(\varrho_{1}, \varrho_{2}, \ldots, \varrho_{n}\right)$ is an inner coupling matrix of the networks that satisfies $\varrho_{j}>0, j=1,2, \ldots, n$. Here, $A^{(r(t))}=$ $\left[a_{i j}^{(r(t))}\right] \in \mathbf{M}_{1}$ and $B^{(r(t))}=\left[b_{i j}^{(r(t))}\right] \in \mathbf{M}_{\mathbf{1}}$ are the outer coupling matrices of the network at time $t . \tau(t)$ is the inner time-varying delay satisfying $\tau \geq \tau(t) \geq 0$ and $\tau_{c}(t)$ is the coupling time-varying delay satisfying $\tau_{c} \geq$ $\tau_{c}(t) \geq 0$. Finally, $\sigma_{i}\left(t, x(t), x(t-\tau(t)), x\left(t-\tau_{c}(t)\right), r(t)\right)=$ $\sigma_{i}\left(t, x_{1}(t), \ldots, x_{n}(t), x_{1}(t-\tau(t)), \ldots, x_{n}(t-\tau(t)), x_{1}(t-\right.$ $\left.\left.\tau_{c}(t)\right), \ldots, x_{n}\left(t-\tau_{c}(t)\right), r(t)\right) \in \mathbb{R}^{n \times n}$ and $w_{i}(t)=\left(w_{i 1}(t)\right.$, $\left.w_{i 2}(t), \ldots, w_{i n}(t)\right)^{T} \in \mathbb{R}^{n}$ is a bounded vector-form Wiener process, satisfying

$$
\begin{gathered}
\mathbb{E}\left[w_{i j}(t)\right]=0, \quad \mathbb{E}\left[w_{i j}^{2}(t)\right]=1, \\
\mathbb{E}\left[w_{i j}(t) w_{i j}(s)\right]=0 \quad(s \neq t) .
\end{gathered}
$$

Suppose that $r(t)(t>0)$ is a right-continuous Markov chain on a probability space that takes on values in a finite space $S=1,2, \ldots, M$ whose generator $\Gamma=\left[\gamma_{i j}\right] \in \mathbb{R}^{M \times M}$ is given by

$$
P\{r(t+\Delta)=j \mid r(t)=i\}= \begin{cases}\gamma_{i j} \Delta+o(\Delta) & \text { if } i \neq j, \\ 1+\gamma_{i i} \Delta+o(\Delta) & \text { if } i=j,\end{cases}
$$

for some $\Delta>0$, where $\gamma_{i j}=0$ is the transition speed from $i$ to $j$ if $i \neq j$ and $\gamma_{i i}=-\sum_{i \neq j} \gamma_{i j}$. By the way, this writing requires that $A^{(r(t))}$ is irreducible. by

The following are some initial conditions for (2) described

$$
x_{i}(s)=\xi_{i}(s), \quad-\check{\tau} \leq s \leq 0, i=1,2, \ldots, N,
$$

where $\check{\tau}=\max \left\{\tau(t), \tau_{c}(t)\right\}, \xi_{i} \in C_{\mathscr{F}_{0}}^{b}\left([-\check{\tau}, 0], \mathbb{R}^{n}\right)$ with the norm $\left\|\xi_{i}\right\|^{2}=\sup _{-\check{\tau} \leq s \leq 0} \xi_{i}(s)^{T} \xi_{i}(s)$.

In this paper, we will make use of the impulse pinning controllers as follows:

$$
\begin{aligned}
\Delta x_{i}\left(t_{k}\right) & =x_{i}\left(t_{k}^{+}\right)-x_{i}\left(t_{k}^{-}\right)=\epsilon_{i k} x_{i}\left(t_{k}^{-}\right)-s\left(t_{k}\right), \\
t & =t_{k}, \quad k \in Z^{+}, \quad i=1,2, \ldots, N,
\end{aligned}
$$

where $\epsilon_{i k}$ are constants and $l<N$.

It is worth noting that system (2) achieves synchronization, that is, $x_{i}(t)=s_{\tilde{i}}(t)$, which implies that we have the following synchronized state equation:

$$
d s_{\check{i}}(t)=f_{\check{i}}\left(t, s_{\tilde{i}}(t), s_{\check{i}}(t-\tau(t))\right) d t .
$$

Let $e_{i}(t)=x_{i}(t)-s_{i}(t)(i=1,2, \ldots, N)$ be the synchronization errors. Then, the error system according to controller (6) can be written as

$$
\begin{array}{r}
d e_{i}(t)=\left\{f_{\tilde{i}}\left(t, x_{i}(t), x_{i}(t-\tau(t))\right)-f_{\tilde{i}}\left(t, s_{\tilde{i}}(t), s_{\tilde{i}}(t-\tau(t))\right)\right. \\
\left.+\sum_{j=1, i \neq j}^{N} a_{i j}^{(r(t))} \sum e_{j}(t)+\sum_{j=1}^{N} b_{i j}^{(r(t))} \sum e_{j}\left(t-\tau_{c}(t)\right)\right\} d t \\
+\sigma_{i}^{r(t)}\left(t, e(t), e(t-\tau(t)), e\left(t-\tau_{c}(t)\right)\right) d w_{i}(t), \\
t \neq t_{k}, \quad k \in Z^{+}, \quad i=1,2, \ldots, N .
\end{array}
$$

$\Delta e_{i}\left(t_{k}\right)=\epsilon_{i k} e_{i}\left(t_{k}^{-}\right), \quad t=t_{k}, k \in Z^{+}, i=1,2, \ldots, N$.

Definition 5. The complex network (2) is said to reach cluster synchronization when the trivial solution of system (8) satisfies the inequality

$$
\sum_{i=1}^{N} \mathbb{E}\left\|e_{i}\left(t, t_{0}, \xi_{i}\right)\right\|^{2} \leq K e^{-\kappa t},
$$

for some positive constants $K$ and $\kappa$ under any initial data $\xi_{i} \in \mathscr{C}_{\mathscr{F}_{0}}^{b}\left([-\tau, 0] ; \mathbb{R}^{n}\right)$.

Definition 6 (see $[21,25])$. A continuous function $f_{\tilde{i}}(t, x, y)$ : $[0,+\infty] \times \mathbb{R}^{n} \times \mathbb{R}^{n} \rightarrow \mathbb{R}^{n}$ is said to belong to the function class QUAD. denoted by $f \in \operatorname{QUAD}\left(P, \Delta, \eta_{\bar{i}}, \zeta_{\bar{i}}\right)$, for some given matrix $\Sigma=\operatorname{diag}\left\{\varrho_{1}, \varrho_{2}, \ldots, \varrho_{n}\right\}$, if there exist a positivedefinite diagonal matrix $P=\operatorname{diag}\left\{p_{1}, p_{2}, \ldots, p_{n}\right\}$, a diagonal matrix $\Delta=\operatorname{diag}\left\{\delta_{1}, \delta_{2}, \ldots, \delta_{n}\right\}$, and constants $\eta_{i}>0, \zeta_{i}>0$ such that $f_{\tilde{i}}(\cdot)$ satisfies the condition

$$
\begin{gathered}
(x-y)^{T} P\left(\left(f_{\tilde{i}}(t, x, z)-f_{\tilde{i}}(t, y, w)\right)-\Delta \Sigma(x-y)\right) \\
\leq-\eta_{\check{i}}(x-y)^{T}(x-y)+\zeta_{\grave{i}}(z-w)^{T}(z-w)
\end{gathered}
$$

for all $x, y, z, w \in \mathbb{R}^{n}$.

Remark 7 (see [25]). The function class QUAD includes almost all the well-known chaotic systems with or without 
delays such as the Lorenz system, the Rössler system, the Chen system, the delayed Chua circuit, the logistic delayed differential system, the delayed Hopfield neural network, and the delayed CNNs.

The following assumptions will be used to establish the synchronization conditions.

(H1) $\tau(t)$ and $\tau_{c}(t)$ are bounded and continuously differentiable functions such that $0<\tau(t) \leq \tau, \dot{\tau}(t)<\bar{\tau}<1$, $0<\tau_{c}(t) \leq \tau_{c}$, and $\dot{\tau}_{c}(t)<\bar{\tau}_{c}<1$. Let $\check{\bar{\tau}}=\max \left\{\bar{\tau}, \bar{\tau}_{c}\right\}$.

(H2) Suppose that $\sigma\left(t, e(t), e(t-\tau(t)), e\left(t-\tau_{c}(t)\right), r\right)=$ $\sigma\left(t, e_{1}(t), \ldots, e_{N}(t), e_{1}(t-\tau(t)), \ldots, e_{N}(t-\tau(t)), e_{1}(t-\right.$ $\left.\left.\tau_{c}(t)\right), \ldots, e_{N}\left(t-\tau_{c}(t)\right), r\right)$. Thereby, there are some positive-definite constant matrices $Y_{i 1}^{r}, \Upsilon_{i 2}^{r}$, and $Y_{i 3}^{r}$ for $i=1,2, \ldots, N$ and $r=1,2, \ldots, M$ such that

$$
\begin{aligned}
& \operatorname{Tr}\left[\sigma_{i}\left(t, e(t), e(t-\tau(t)), e\left(t-\tau_{c}(t)\right), r\right)^{T}\right. \\
& \left.\quad \times \sigma_{i}\left(t, e(t), e(t-\tau(t)), e\left(t-\tau_{c}(t)\right), r\right)\right] \\
& \leq \sum_{j=1}^{N} e_{j}(t)^{T} \Upsilon_{i 1}^{r} e_{j}(t)+\sum_{j=1}^{N} e_{j}(t-\tau(t))^{T} \Upsilon_{i 2}^{r} e_{j}(t-\tau(t)) \\
& \quad+\sum_{j=1}^{N} e_{j}\left(t-\tau_{c}(t)\right)^{T} \Upsilon_{i 3}^{r} e_{j}\left(t-\tau_{c}(t)\right) .
\end{aligned}
$$

Lemma 8 (see [26]). Consider a stochastic delayed differential equation with Markovian switching expressed as

$$
\begin{aligned}
d x(t)= & f(t, x(t), x(t-\tau), r(t)) d t \\
& +\sigma(t, x(t), x(t-\tau), r(t)) d \omega(t)
\end{aligned}
$$

on $t \geq 0$ with initial value $x_{0}=\xi \in C_{F_{0}}^{b}\left([-\tau, 0] ; \mathbb{R}^{n}\right)$, where

$$
f: \mathbb{R}^{n} \times \mathbb{R}_{+} \times S \longrightarrow \mathbb{R}^{n}, \quad \sigma: \mathbb{R}^{n} \times \mathbb{R}_{+} \times S \longrightarrow \mathbb{R}^{n \times m} .
$$

Let $C^{2,1}\left(\mathbb{R}_{+} \times \mathbb{R}^{n} ; \mathbb{R}_{+}\right)$be the family of all the nonnegative functions $V(t, x, r)$ on $\mathbb{R}_{+} \times \mathbb{R}^{n} \times S$ which are twice continuously differentiable in $x$ and once differentiable in $t$. Let $V \in$ $C^{2,1}\left(\mathbb{R}_{+} \times \mathbb{R}^{n} \times S ; \mathbb{R}_{+}\right)$. Define an operator $\mathscr{L} V$ from $\mathbb{R}^{n} \times \mathbb{R}_{+} \times S$ to $\mathbb{R}^{n}$ by

$$
\begin{aligned}
\mathscr{L} V(t, x, r)= & V_{t}(t, x, r)+V_{x}(t, x, r) f(t, x, r) \\
& +\frac{1}{2} \operatorname{Tr}\left[\sigma(t, x, r)^{T} V_{x x} \sigma(t, x, r)\right] \\
& +\sum_{j=1}^{M} \gamma_{i j} V(t, x, j),
\end{aligned}
$$

where $V_{t}(t, x, r)=\partial V(t, x, r) / \partial t, V_{x}(t, x, r)=(\partial V(t, x, r) /$ $\left.\partial x_{1}, \ldots, \partial V(t, x, r) / \partial x_{n}\right)$, and $V_{x x}(t, x, r)=\left(\partial^{2} V(t, x, r) / \partial x_{i}\right.$ $\left.x_{j}\right)_{n \times n}$. If $V \in C^{2,1}\left(\mathbb{R}_{+} \times \mathbb{R}^{n} \times S ; \mathbb{R}_{+}\right)$, then

$\mathbb{E} V(t, x(t), r)=\mathbb{E} V\left(t_{0}, x\left(t_{0}\right), r\right)+\mathbb{E} \int_{t_{0}}^{t} \mathscr{L} V(s, x(s), r) d s$ for all $\infty>t>t_{0} \geq 0$ as long as the expectations of the integrals exist.

\section{Main Result}

This section will show our main results with rigorous proof.

Theorem 9. Suppose that (H1) and (H2) are true and $f_{\check{i}} \in$ $\operatorname{QUAD}\left(P, \Delta, \eta_{\check{i}}, \zeta_{\check{i}}\right)$. If there are some positive constants $\theta, \alpha_{r}$, and $\beta_{r}$ such that

$$
\begin{gathered}
{\left[\begin{array}{cc}
A(r)^{s}+\check{\delta} I_{N}-\alpha_{r} I_{N} & \frac{B(r)}{2} \\
\frac{B(r)^{T}}{2} & -\beta_{r} I_{N}
\end{array}\right] \leq 0, \quad \text { for } r=1,2, \ldots, M} \\
0 \leq \check{\bar{\tau}} \leq 1-\frac{\check{q}(\check{b}+\check{c})}{1+\theta} \\
\varphi(\check{\tau}+T)+2 \ln \frac{\check{q}}{\widehat{q}}\left|1+\epsilon_{v}\right|-\gamma T<0 \\
\left(\frac{1}{b_{1}+c_{1}}, \frac{1}{b_{2}+c_{2}}, \ldots, \frac{1}{b_{M}+c_{M}}\right)^{T}>\widetilde{\Gamma}^{-1} \mathbf{1}_{M}
\end{gathered}
$$

where

$$
\begin{aligned}
\varphi & =1+\theta+\gamma \check{q}_{r}+\frac{\check{b} \check{q}}{1-\bar{\tau}} e^{\gamma \tau}+\frac{\check{c} \check{q}}{1-\bar{\tau}_{c}} e^{\gamma \tau_{c}}, \\
\widetilde{\Gamma} & =\operatorname{diag}\left\{a_{1}, a_{2}, \ldots, a_{M}\right\}+\Gamma, \\
a_{r} & =\frac{\lambda_{\max }\left(-2 \eta_{i} I_{N}+\check{p} \sum_{j=1}^{N} \Upsilon_{j 1}^{r}+2 \alpha_{r} P \Sigma\right)}{\check{p}}, \quad \check{a}=\max _{r \in S, i=1, \ldots, N} a_{r}, \\
b_{r} & =\frac{\lambda_{\max }\left(\sum_{j=1}^{N} P \Upsilon_{j 2}^{r}+2 \zeta_{\check{i}} I_{N}\right)}{\widehat{p}}, \quad \check{b}=\max _{r \in S, i=1, \ldots, N} b_{r}, \\
c_{r}= & \frac{\lambda_{\max }\left(\sum_{j=1}^{N} P \Upsilon_{j 3}^{r}+2 \beta_{r} P \Sigma\right)}{\widehat{p}}, \quad \check{c}=\max _{r \in S} c_{r},
\end{aligned}
$$

then the global and exponential synchronization of the stochastic complex network (8) can be achieved.

Proof. Inequality (19) indicates that there is a sufficiently small constant $\theta>0$ such that

$$
\left(\frac{1}{b_{1}+c_{1}}, \frac{1}{b_{2}+c_{2}}, \ldots, \frac{1}{b_{M}+c_{M}}\right)^{T} \geq(1+\theta) \widetilde{\Gamma}^{-1} \mathbf{1} .
$$

Let $(1+\theta) \widetilde{\Gamma}^{-1} \mathbf{1}=q=\left(q_{1}, q_{2}, \ldots, q_{M}\right)^{T}$. Thus

$$
\widetilde{\Gamma} q=(1+\theta) \mathbf{1}_{M}
$$

namely,

$$
\left(b_{r}+c_{r}\right) q_{r} \leq 1 \quad \text { while } a_{r} q_{r}+\sum_{s=1}^{M} \gamma_{r s} q_{s}=1+\theta .
$$




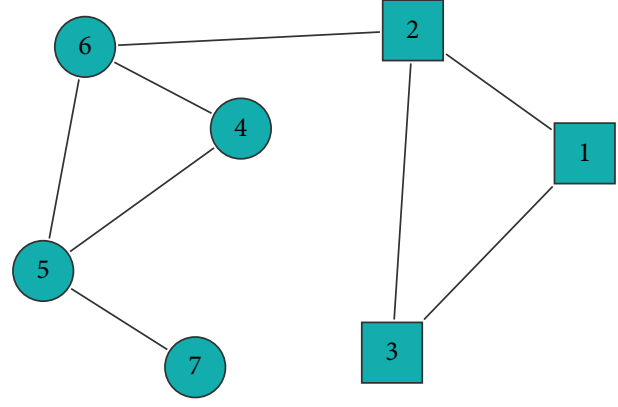

(a)

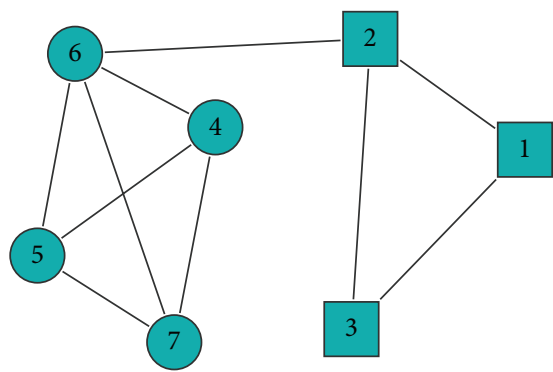

(c)

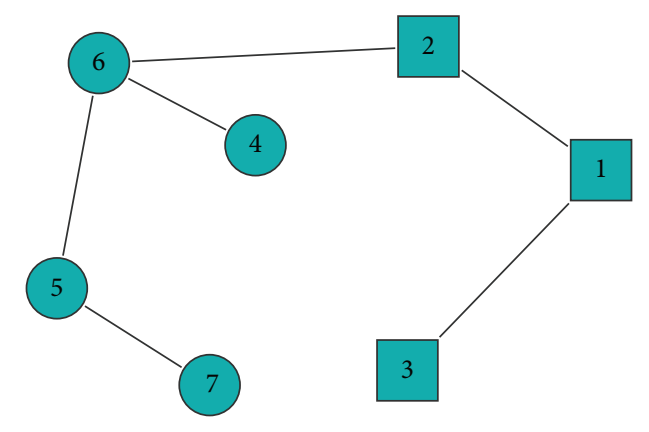

(b)

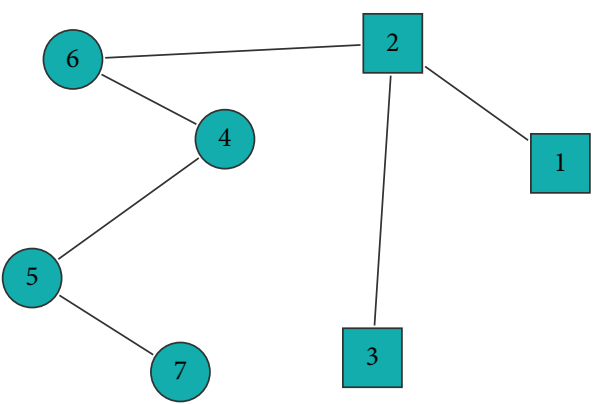

(d)

FIGURE 1: The topology structures of the switching networks that have 7 nodes divided into 2 clusters (nodes $\{1,2,3\}$ and nodes $\{4,5,6,7\}$ ). (a) and (c) show the topology structures of the coupling matrices $A(1)$ and $A(2)$, respectively; (b) and (d) express the topology structures of the coupling matrices $B(1)$ and $B(2)$, respectively.

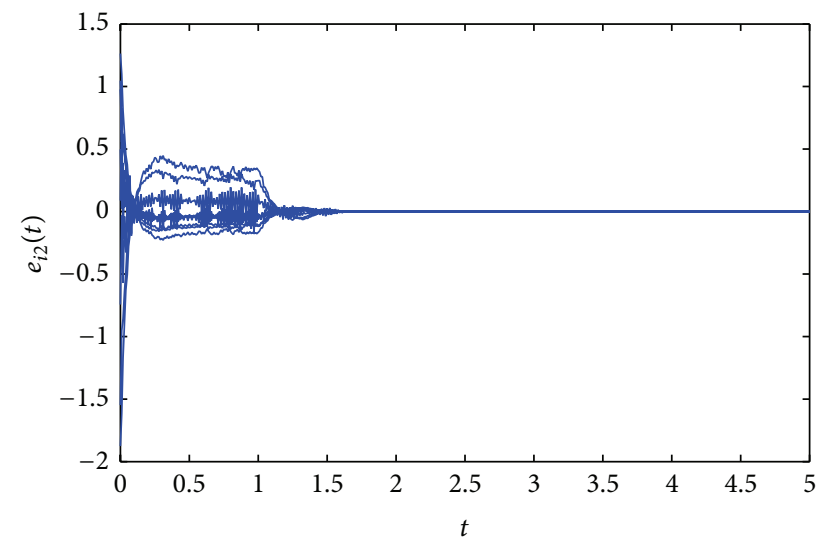

(a)

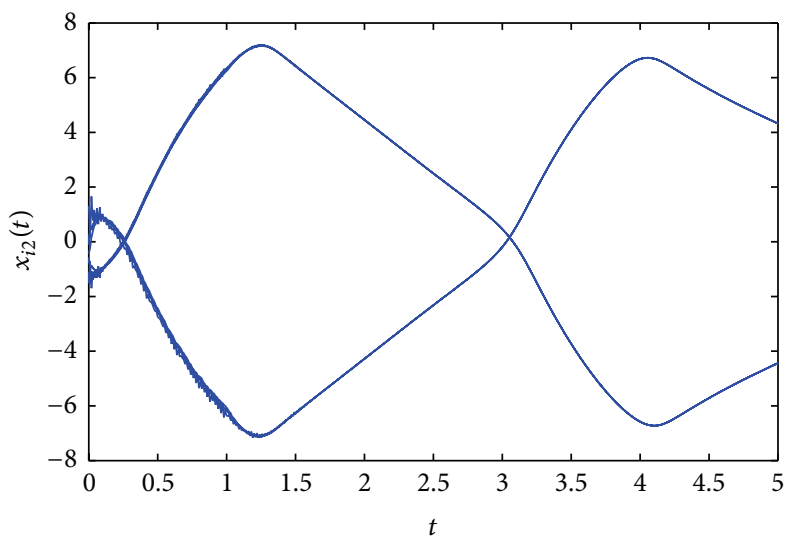

(b)

FIGURE 2: The trajectories of the state variables of $x_{i 1}$ and $x_{i 2}(i=1,2, \ldots, 7)$ in system (46) by impulse control.

For $1 \leq r \leq M$, define the Lyapunov-Krasovskii function

$$
V(t, e(t), r)=\frac{1}{2} q_{r} \sum_{i=1}^{N} e_{i}(t)^{T} P e_{i}(t)
$$

and let $e^{-k}(t)=\left(e_{1 k}(t), e_{2 k}(t), \ldots, e_{N k}(t)\right)^{T}, k=1,2, \ldots, n$. By Lemma 8 , for any $t \in\left[t_{k-1}, t_{k}\right), k=1,2, \ldots$, we have $\mathscr{L} V(t, e(t), r)$

$$
=q_{r} \sum_{i=1}^{N} e_{i}(t)^{T} P\left\{f_{\check{i}}\left(t, x_{i}(t), x_{i}(t-\tau(t))\right)\right.
$$

$$
\begin{aligned}
& -f_{\check{i}}\left(t, s_{\check{i}}(t), s_{\check{i}}(t-\tau(t))\right) \\
& +\sum_{j=1}^{N} a_{i j}(r) \sum e_{j}(t)
\end{aligned}
$$$$
\left.+\sum_{j=1}^{N} b_{i j}(r) \Sigma e_{j}\left(t-\tau_{c}(t)\right)\right\}
$$$$
+\frac{1}{2} q_{r} \sum_{i=1}^{N} \operatorname{Tr}\left\{\sigma_{i}\left(t, x(t), x(t-\tau(t)), x\left(t-\tau_{c}(t)\right), r\right)^{T}\right.
$$ 


$$
\begin{aligned}
& \left.\times P \sigma_{i}\left(t, x(t), x(t-\tau(t)), x\left(t-\tau_{c}(t)\right), r\right)\right\} \\
& +\sum_{s=1}^{M} \gamma_{r s} q_{s} \frac{1}{2} \sum_{i=1}^{N} e_{i}(t)^{T} P e_{i}(t) \\
& \leq q_{r}\left\{-\sum_{i=1}^{N} \eta_{i} e_{i}(t)^{T} e_{i}(t)+\sum_{i=1}^{N} \zeta_{i} e_{i}(t-\tau(t))^{T} e_{i}(t-\tau(t))\right. \\
& +\sum_{k=1}^{n} p_{k} \varrho_{k} \delta_{k} e^{-k}(t)^{T} e^{-k}(t)+\sum_{k=1}^{n} p_{k} \varrho_{k} e^{-k}(t)^{T} A(r) e^{-k}(t) \\
& +\sum_{k=1}^{n} p_{k} \varrho_{k} e^{-k}(t)^{T} B(r) e^{-k}\left(t-\tau_{c}(t)\right) \\
& +\frac{1}{2} \check{p} \sum_{j=1}^{N}\left[\sum_{i=1}^{N} e_{i}(t)^{T} \Upsilon_{j 1}^{r} e_{i}(t)\right. \\
& +\sum_{i=1}^{N} e_{i}(t-\tau(t))^{T} \Upsilon_{j 2}^{r} e_{i}(t-\tau(t)) \\
& \left.\left.+\sum_{i=1}^{N} e_{i}\left(t-\tau_{c}(t)\right)^{T} \Upsilon_{j 3}^{r} e_{i}\left(t-\tau_{c}(t)\right)\right]\right\} \\
& +\sum_{s=1}^{M} \gamma_{r s} q_{s} \frac{1}{2} \sum_{i=1}^{N} e_{i}(t)^{T} P e_{i}(t) \\
& =q_{r}\left\{\sum_{i=1}^{N} e_{i}(t)^{T}\left(-\eta_{i} I_{N}+\frac{1}{2} \check{p} \sum_{j=1}^{N} \Upsilon_{j 1}^{r}+\alpha_{r} P \Sigma\right) e_{i}(t)\right. \\
& +\sum_{i=1}^{N} e_{i}(t-\tau(t))^{T}\left(\zeta_{\check{i} I_{N}}+\frac{1}{2} \check{p} \sum_{j=1}^{N} \Upsilon_{j 2}^{r}\right) e_{i}(t-\tau(t)) \\
& +\sum_{i=1}^{N} e_{i}\left(t-\tau_{c}(t)\right)^{T}\left(\frac{1}{2} \check{p} \sum_{j=1}^{N} \Upsilon_{j 3}^{r}+\beta_{r} P \Sigma\right) \\
& \left.\times e_{i}\left(t-\tau_{c}(t)\right)\right\} \\
& +\sum_{s=1}^{M} \gamma_{r s} q_{s} \frac{1}{2} \sum_{i=1}^{N} e_{i}(t)^{T} P e_{i}(t) \\
& +q_{r}\left\{\sum_{k=1}^{n} p_{k} \varrho_{k} \bar{e}^{-k}(t)^{T}\left[A(r)+\left(\check{\delta}-\alpha_{r}\right) I_{N}\right] \bar{e}^{k}(t)\right. \\
& +\sum_{k=1}^{n} p_{k} \varrho_{k} e^{-k}(t)^{T} B(r) e^{-k}\left(t-\tau_{c}(t)\right) \\
& \left.-\sum_{k=1}^{n} p_{k} \varrho_{k} e^{-k}\left(t-\tau_{c}(t)\right)^{T} \beta_{r} e^{-k}\left(t-\tau_{c}(t)\right)\right\} \\
& \leq q_{r}\left\{\sum_{i=1}^{N} e_{i}(t)^{T}\left(-\eta_{i} I_{N}+\frac{1}{2} \check{p} \sum_{j=1}^{N} \Upsilon_{j 1}^{r}+\alpha_{r} P \Sigma\right) e_{i}(t)\right.
\end{aligned}
$$

Set

$$
E(t)=\frac{1}{2} \sum_{i=1}^{N} e_{i}(t)^{T} P e_{i}(t)
$$

And then we have

$$
\begin{aligned}
\mathscr{L} V(t) \leq & a_{r} q_{r} E(t)+b_{r} q_{r} E(t-\tau(t))+c_{r} q_{r} E\left(t-\tau_{c}(t)\right) \\
& +\sum_{s=1}^{M} \gamma_{r s} q_{s} E(t)
\end{aligned}
$$

and (23) deduces that

$$
\mathscr{L} V(t) \leq(1+\theta) E(t)+\check{b} \check{q} E(t-\tau(t))+\check{c} \check{q} E\left(t-\tau_{c}(t)\right)
$$

Define

$$
W(t)=e^{\gamma t} V(t)
$$

By the aid of (28), we will deal with the operator

$$
\begin{aligned}
& \mathscr{L} W(t)=e^{\gamma t}[\gamma V(t)+\mathscr{L} V(t)] \\
& \leq e^{\gamma t}[\gamma \check{q} E(t)+(1+\theta) E(t)+\check{b} \check{q} E(t-\tau(t)) \\
&\left.+\check{c} \check{q} E\left(t-\tau_{c}(t)\right)\right] .
\end{aligned}
$$

The generalized Itô formula shows that

$$
e^{\gamma t} \mathbb{E} V(t)=e^{\gamma t_{0}} \mathbb{E} V\left(t_{0}\right)+\mathbb{E} \int_{t_{0}}^{t} \mathscr{L} W(s) d s
$$

for any $t_{k}>t>t_{0}>t_{k-1} \geq 0$. Hence we have

$$
\begin{aligned}
e^{\gamma t} \mathbb{E} V(t) \leq & e^{\gamma t_{0}} \mathbb{E} V\left(t_{0}\right) \\
+ & \mathbb{E} \int_{t_{0}}^{t} e^{\gamma s}[\gamma \check{q} E(s)+(1+\theta) E(s) \\
& \left.+\check{b} \check{q} E(s-\tau(s))+\check{c} \check{q} E\left(s-\tau_{c}(s)\right)\right] d s
\end{aligned}
$$




$$
\begin{aligned}
\leq & \check{q} e^{\gamma t_{0}} \mathbb{E} E\left(t_{0}\right)+(\gamma \check{q}+1+\theta) \int_{t_{0}}^{t} e^{\gamma s} \mathbb{E} E(s) d s \\
& +\check{b} \check{q} e^{\gamma \tau} \int_{t_{0}}^{t} e^{\gamma(s-\tau(s))} \mathbb{E} E(s-\tau(s)) d s \\
& +\check{c} \check{q} e^{\gamma \tau_{c}} \int_{t_{0}}^{t} e^{\gamma\left(s-\tau_{c}(s)\right)} \mathbb{E} E\left(s-\tau_{c}(s)\right) d s .
\end{aligned}
$$

Let $s-\tau(s)=u$; we have

$$
\begin{aligned}
\int_{t_{0}}^{t} e^{\gamma(s-\tau(s))} \mathbb{E} E(s-\tau(s)) d s & =\int_{t_{0}-\tau\left(t_{0}\right)}^{t-\tau(t)} e^{\gamma u} \mathbb{E} E(u) \frac{d u}{1-\dot{\tau}(t)} \\
& \leq \frac{1}{1-\bar{\tau}} \int_{t_{0}-\tau}^{t} e^{\gamma u} \mathbb{E} E(u) d u
\end{aligned}
$$

Similarly, it can be obtained that

$$
\int_{t_{0}}^{t} e^{\gamma\left(s-\tau_{c}(s)\right)} \mathbb{E} E(s-\tau(s)) d s \leq \frac{1}{1-\bar{\tau}_{c}} \int_{t_{0}-\tau_{c}}^{t} e^{\gamma u} \mathbb{E} E(u) d u .
$$

Substituting (33) and (34) into (32) can result in

$$
e^{\gamma t} \mathbb{E} V(t) \leq q_{r} e^{\gamma t_{0}} \mathbb{E} V\left(t_{0}\right)+\varphi \int_{t_{0}-\check{\tau}}^{t} e^{\gamma u} \mathbb{E} E(u) d u
$$

With help of Gronwall inequality, the following inequality can be achieved:

$$
\mathbb{E} E(t) \leq \frac{\check{q}}{\widehat{q}} \mathbb{E} E\left(t_{0}\right) e^{\varphi\left(t-t_{0}+\check{\tau}\right)+\gamma\left(t_{0}-t\right)} .
$$

On the other hand, in view of the construction of $E(t)$, we have

$$
E\left(t_{k}\right) \leq\left(1+\epsilon_{k}\right)^{2} E\left(t_{k}^{-}\right)
$$

where $\left|1+\epsilon_{k}\right|=\max _{i=1,2, \ldots, N}\left|1+\epsilon_{i k}\right|$.

Let $k=\left\lfloor\left(t-t_{0}\right) / T\right\rfloor$; according to (36) and (37), for any $t \in\left[t_{k-1}, t_{k}\right)$, one has

$$
\begin{aligned}
\mathbb{E}[V(t)] & \leq \frac{\check{q}}{\hat{q}} \mathbb{E} E\left(t_{k}\right) e^{\varphi\left(t-t_{k}+\check{\tau}\right)+\gamma\left(t_{k}-t\right)} \\
& \leq \frac{\check{q}}{\widehat{q}} \mathbb{E} V\left(t_{k-1}^{-}\right) e^{\varphi\left(t-t_{k-1}+\check{\tau}\right)+\gamma\left(t_{k}-t\right)+2 \ln \left|1+\epsilon_{k-1}\right|} \\
& \leq \cdots \leq\left(\frac{\check{q}}{\hat{q}}\right)^{k-1} \mathbb{E} E(0) e^{\varphi(t+k \check{\tau})-\gamma t+\sum_{v=1}^{k-1} 2 \ln \left|1+\epsilon_{v}\right|} .
\end{aligned}
$$

Let $|1+\epsilon|=\max _{v \in Z^{+}}\left|1+\epsilon_{v}\right|$; we have

$$
\mathbb{E}[E(t)] \leq \mathbb{E} E(0) e^{\varphi(t+k \check{\tau})-\gamma t+2(k-1) \ln (\check{q} / \widehat{q})|1+\epsilon|} .
$$

By means of condition (18) in Theorem 9, there is a number $\eta$ such that $\mathbb{E} E(t) \leq \mathbb{E} E\left(t_{0}\right) e^{-\eta t}$. Consequently, $\mathbb{E}\left\|e_{i}(t)\right\| \leq\left(E\left(t_{0}\right) / \check{p}\right)^{1 / 2} e^{-(\eta / 2)\left(t-t_{0}\right)}$. The proof of Theorem 9 is completed.
When the time-varying delays are constant (i.e., $\tau(t)=\tau$, $\tau_{c}(t)=\tau_{c}$ ), we obtain the following corollary.

Corollary 10. Suppose that (H1) and (H2) are true and $f_{\check{i}} \in$ $\mathrm{QUAD}\left(P, \Delta, \eta_{\tilde{i}}, \zeta_{\check{i}}\right)$. If there are some positive constants $\theta, \alpha_{r}$, and $\beta_{r}$ such that

$$
\begin{gathered}
{\left[\begin{array}{cc}
A(r)^{s}+\check{\delta} I_{N}-\alpha_{r} I_{N} & \frac{B(r)}{2} \\
\frac{B(r)^{T}}{2} & -\beta_{r} I_{N}
\end{array}\right] \leq 0, \quad \text { for } r=1,2, \ldots, M,} \\
\varphi(\check{\tau}+T)+2 \ln \frac{\check{q}}{\widehat{q}}\left|1+\epsilon_{v}\right|-\gamma T<0, \\
\left(\frac{1}{b_{1}+c_{1}}, \frac{1}{b_{2}+c_{2}}, \ldots, \frac{1}{b_{M}+c_{M}}\right)^{T}>\widetilde{\Gamma}^{-1} \mathbf{1}_{M},
\end{gathered}
$$

where

$$
\begin{aligned}
& \varphi=1+\theta+\gamma \check{q}_{r}+\check{b} \check{q} e^{\gamma \tau}+\check{c} \check{q} e^{\gamma \tau_{c}}, \\
& \widetilde{\Gamma}=\operatorname{diag}\left\{a_{1}, a_{2}, \ldots, a_{M}\right\}+\Gamma, \\
& a_{r}=\frac{\lambda_{\max }\left(-2 \eta_{i} I_{N}+\check{p} \sum_{j=1}^{N} \Upsilon_{j 1}^{r}+2 \alpha_{r} P \Sigma\right)}{\check{p}}, \quad \check{a}=\max _{r \in S, i=1, \ldots, N} a_{r}, \\
& b_{r}=\frac{\lambda_{\max }\left(\sum_{j=1}^{N} P \Upsilon_{j 2}^{r}+2 \zeta_{\check{i}} I_{N}\right)}{\widehat{p}}, \quad \check{b}=\max _{r \in S, i=1, \ldots, N} b_{r}, \\
& c_{r}=\frac{\lambda_{\max }\left(\sum_{j=1}^{N} P \Upsilon_{j 3}^{r}+2 \beta_{r} P \Sigma\right)}{\widehat{p}}, \quad \check{c}=\max _{r \in S} c_{r},
\end{aligned}
$$

then the global and exponential synchronization of the stochastic complex network (8) can be achieved.

When $A(r)$ and $B(r)$ are symmetric matrices, for $r=$ $1,2, \ldots, M$, and $\sigma(\cdot)=\mathbf{0}$, we can get the following corollary.

Corollary 11. Suppose that (H1) and (H2) are true and $f_{\check{i}} \in$ $\operatorname{QUAD}\left(P, \Delta, \eta_{\check{i}}, \zeta_{\check{i}}\right)$. If there are some positive constants $\theta, \alpha_{r}$, and $\beta_{r}$ such that

$$
\begin{gathered}
{\left[\begin{array}{cc}
A(r)+\check{\delta} I_{N}-\alpha_{r} I_{N} & \frac{B(r)}{2} \\
\frac{B(r)^{T}}{2} & -\beta_{r} I_{N}
\end{array}\right] \leq 0, \quad \text { for } r=1,2, \ldots, M,} \\
0 \leq \check{\bar{\tau}} \leq 1-\frac{\check{q}(\check{b}+\check{c})}{1+\theta}, \\
\varphi(\check{\tau}+T)+2 \ln \frac{\check{q}}{\widehat{q}}\left|1+\epsilon_{v}\right|-\gamma T<0, \\
\left(\frac{1}{b_{1}+c_{1}}, \frac{1}{b_{2}+c_{2}}, \ldots, \frac{1}{b_{M}+c_{M}}\right)^{T}>\widetilde{\Gamma}^{-1} \mathbf{1}_{M},
\end{gathered}
$$




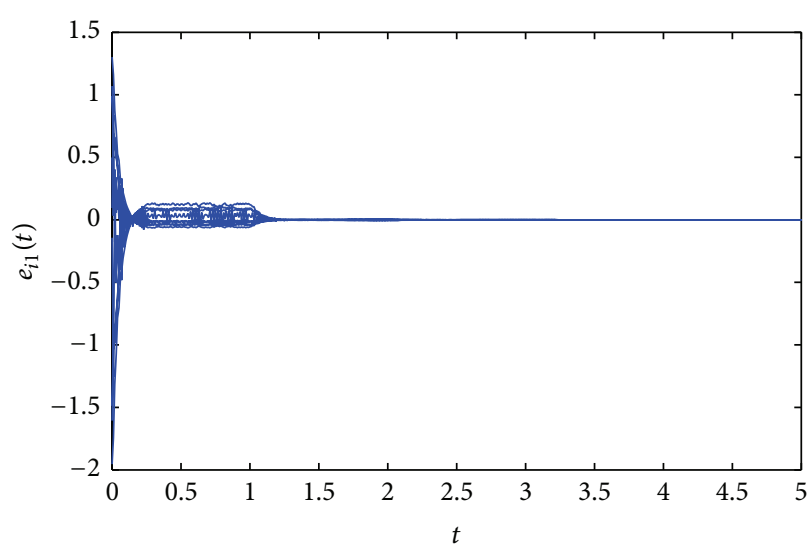

(a)

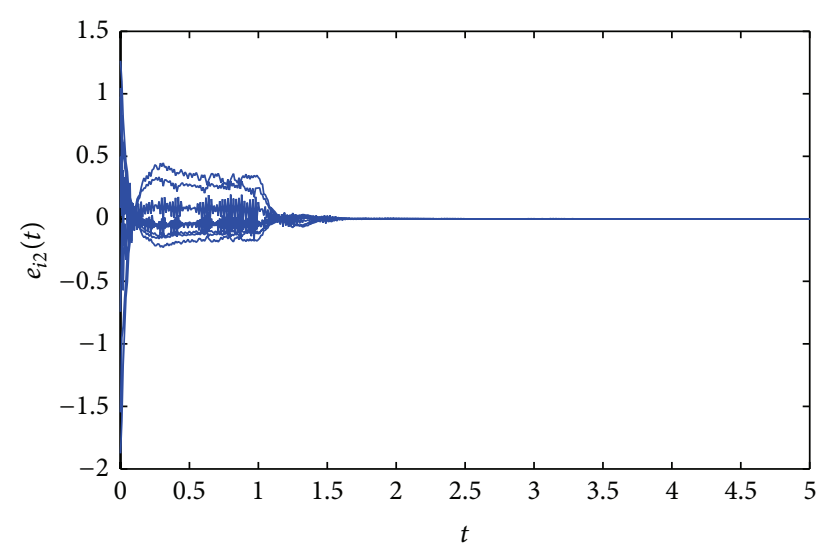

(b)

FIgURE 3: The time evolution of $e_{i 1}$ and $e_{i 2}(i=1,2, \ldots, 7)$ in system (46) by impulse control.

where

$$
\begin{aligned}
\varphi & =1+\theta+\gamma \check{q}_{r}+\frac{\check{b} \check{q}}{1-\bar{\tau}} e^{\gamma \tau}+\frac{\check{c} \check{q}}{1-\bar{\tau}_{c}} e^{\gamma \tau_{c}}, \\
\widetilde{\Gamma} & =\operatorname{diag}\left\{a_{1}, a_{2}, \ldots, a_{M}\right\}+\Gamma, \\
a_{r} & =\frac{\lambda_{\max }\left(-2 \eta_{i} I_{N}+2 \alpha_{r} P \Sigma\right)}{\check{p}}, \quad \check{a}=\max _{r \in S, i=1, \ldots, N} a_{r}, \\
b_{r} & =\frac{2 \zeta_{\check{i}}}{\widehat{p}}, \quad \check{b}=\max _{r \in S, i=1, \ldots, N} b_{r}, \\
c_{r} & =\frac{\lambda_{\max }\left(2 \beta_{r} P \Sigma\right)}{\widehat{p}}, \quad \check{c}=\max _{r \in S} c_{r},
\end{aligned}
$$

then the global and exponential synchronization of the stochastic complex network (8) can be achieved.

\section{Numerical Simulation}

This section will employ some numerical examples to illustrate the effectiveness of the previous theoretical results.

Consider the following stochastic delayed neural network:

$$
\begin{aligned}
d s(t)= & \{-\operatorname{Cs}(t)+\operatorname{Dhf}(s(t))+\operatorname{Eh}(s(t-\tau(t)))\} d t \\
& +\sigma(s(t), s(t-\tau(t))) d w(t)
\end{aligned}
$$

where $h(s)=\tanh (s), \tau(t)=1, \sigma(s(t), s(t-\tau(t)))=$ $\operatorname{diag}\left\{s_{1}(t), s_{2}(t)\right\}$,

$$
\begin{gathered}
C=\left[\begin{array}{ll}
1 & 0 \\
0 & 1
\end{array}\right], \quad D=\left[\begin{array}{cc}
2 & -0.1 \\
-5 & 4.5
\end{array}\right], \\
E=\left[\begin{array}{cc}
-1.5 & -0.1 \\
-0.2 & -4
\end{array}\right] .
\end{gathered}
$$

Set $P=\operatorname{diag}\{1,1\}, \Delta=\operatorname{diag}\{5,11.5\}$, and let $\eta=0.15, \zeta=3.25$ ensure that condition (10) is satisfied. And then

$$
\begin{gathered}
d x_{i}(t)=\left\{f\left(t, x_{i}(t), x_{i}(t-\tau(t))\right)\right. \\
\left.\quad+\sum_{j=1}^{7} a_{i j}^{r} \sum x_{j}(t)+\sum_{j=1}^{7} b_{i j}^{r} \sum x_{j}\left(t-\tau_{c}(t)\right)\right\} d t \\
+\sigma_{i}^{r}\left(t, x(t), x(t-\tau(t)), x\left(t-\tau_{c}(t)\right)\right) d w_{i}(t), \quad i=1,2, \ldots, 5, \quad r=1,2, \\
\Gamma=\left[\begin{array}{cc}
-1 & 1 \\
2 & -2
\end{array}\right], \quad \tau_{c}(t)=0.1 \frac{e^{t}}{1+e^{t}}, \\
\sigma_{i}^{1}\left(t, x(t), x(t-\tau(t)), x\left(t-\tau_{c}(t)\right)\right) \\
=0.1 \operatorname{diag}\left\{x_{i 1}(t)-x_{i+1,1}(t), x_{i 2}(t)-x_{i+1,2}(t)\right\} \\
\sigma_{i}^{2}\left(t, x(t), x(t-\tau(t)), x\left(t-\tau_{c}(t)\right)\right) \\
=0.1 \operatorname{diag}\left\{x_{i 1}(t-\tau(t))-x_{i+1,1}(t-\tau(t))\right. \\
\left.x_{i 2}(t-\tau(t))-x_{i+1,2}(t-\tau(t))\right\}
\end{gathered}
$$

We here consider a network that has 7 nodes divided into 2 clusters as shown in Figure 1. Some computations result in $\tau=1, \bar{\tau}=0, \tau_{c}=0.025, \bar{\tau}_{c}=0.1, \Upsilon_{i j}=0.01 I_{2}$ for $i=$ $1,2, \ldots, N$. Thus, the solutions of inequalities can be obtained as follows (16)-(19): $\alpha_{1}=3.150, \beta_{1}=0.001, a_{1}=2.903, b_{1}=$ 4.207, and $c_{1}=0.089 ; \alpha_{2}=3.806, \beta_{2}=0.012, a_{2}=3.105$, $b_{2}=2.103$, and $c_{2}=0.105$.

In this simulation, the initial conditions, $x_{i j}\left(t_{0}\right)$ for $i=$ $1,2, \ldots, 7, j=1,2$, are all constants. Figure 2 provides the trajectories of the impulse pinning control gains. By Figure 3, we show the time evolution of the cluster synchronization errors with impulse control. 


\section{Conclusion}

In this writing, we focused on the cluster synchronization issue of an array of stochastic complex networks with Markovian switching and time-varying delayed couplings. By means of an impulsive pinning control method imposed on a small fraction of the nodes, the desired cluster synchronization was reached, while a novel sufficient condition was derived to ensure the stability of the considered stochastic networks. At the end of this paper, a numerical simulation was given to show the validity of the theoretical analysis.

\section{Conflict of Interests}

The authors declare that there is no conflict of interests regarding the publication of this paper.

\section{Acknowledgments}

This work was supported by the Basic Research Project Fund (2213K3170015 and 2213K3170021) of Shenzhen Polytechnic, Shenzhen. The authors are very grateful to the reviewers and editor for their valuable comments and suggestions to improve the presentation of the paper.

\section{References}

[1] L. A. N. Amaral, A. Scala, M. Barthélémy, and H. E. Stanley, "Classes of small-world networks," Proceedings of the National Academy of Sciences of the United States of America, vol. 97, pp. 11149-11152, 2000.

[2] A. Zheleznyak and L. O. Chua, "coexistence of low- and highdimensional spatiotemporal chaos in a chain of dissipatively coupled chua's circuits," International Journal of Bifurcation and Chaos, vol. 4, pp. 639-674, 1994.

[3] M. Faloutsos, P. Faloutsos, and C. Faioutsos, "On powerlaw relationships of the internet topology," in Proceedings of the ACM SIGCOMM Conference Applications, Technologies, Architectures, and Protocols for Computer Communication, pp. 251-261, September 1999.

[4] A. Medina, I. Matt, and J. Byers, "On the origin of power laws in internet topologies," Computer Communication Review, vol. 30, pp. 18-28, 2000.

[5] A. Perez-Munuzuri, V. Perez-Munuzuri, V. Perez-Villar, and L. O. Chua, "Synchronization in an array of linearly coupled dynamical systems," IEEE Transactions on Circuits and Systems I: Fundamental Theory and Applications, vol. 42, no. 8, pp. 430447, 1995.

[6] R. Albert, H. Jeong, and A. L. Barabási, "Diameter of the worldwide web," Nature, vol. 401, no. 6749, pp. 130-131, 1999.

[7] S. H. Wang, J. Y. Kuang, J. H. Li, and Y. L. Luo, "Chaos-based secure communications in a large community," Physical Review E, vol. 66, Article ID 065202, 2002.

[8] B. Rakshit, A. R. Chowdhury, and P. Saha, "Parameter estimation of a delay dynamical system using synchronization in presence of noise," Chaos, Solitons and Fractals, vol. 32, no. 4, pp. 1278-1284, 2007.

[9] Z. G. Zheng and G. Hu, "Generalized synchronization versus phase synchronization," Physical Review E, vol. 62, article 7882, 2000 .
[10] M. G. Earl and S. H. Strogatz, "Synchronization in oscillator networks with delayed coupling: A stability criterion," Physical Review E, vol. 67, no. 3, Article ID 036204, 2003.

[11] E. G. de Oliveira and T. Braun, "Partial synchronization on a network with different classes of oscillators," Physical Review E, vol. 76, Article ID 067201, 2007.

[12] K. Wang, X. Fu, and K. Li, "Cluster synchronization in community networks with nonidentical nodes," Chaos. An Interdisciplinary Journal of Nonlinear Science, vol. 19, no. 2, Article ID 023106, 2009.

[13] K. Kaneko, "Relevance of dynamic clustering to biological networks," Physica D: Nonlinear Phenomena, vol. 75, no. 1-3, pp. 55-73, 1994.

[14] I. Belykh, V. Belykh, K. Nevidin, and M. Hasler, "Persistent clusters in lattices of coupled nonidentical chaotic systems," Chaos, vol. 13, no. 1, pp. 165-178, 2003.

[15] A. Pogromsky, G. Santoboni, and H. Nijmeijer, "Partial synchronization: from symmetry towards stability," Physica D, vol. 172, no. 1-4, pp. 65-87, 2002.

[16] Z. Ma, Z. Liu, and G. Zhang, "A new method to realize cluster synchronization in connected chaotic networks," Chaos, vol. 16, no. 2, Article ID 023103, 2006.

[17] Y. Sun, J. Cao, and Z. Wang, "Exponential synchronization of stochastic perturbed chaotic delayed neural networks," Neurocomputing, vol. 70, no. 13-15, pp. 2465-2477, 2007.

[18] J. Cao, Z. Wang, and Y. Sun, "Synchronization in an array of linearly stochastically coupled networks with time delays," Physica A, vol. 385, no. 2, pp. 718-728, 2007.

[19] X. Yang and J. Cao, "Stochastic synchronization of coupled neural networks with intermittent control," Physics Letters A, vol. 373, no. 36, pp. 3259-3272, 2009.

[20] I. V. Belykh, V. N. Belykh, and M. Hasler, "Blinking model and synchronization in small-world networks with a time-varying coupling," Physica D, vol. 195, no. 1-2, pp. 188-206, 2004.

[21] J. Wang, C. Xu, J. Feng, M. K. Kwong, and F. Austin, "Meansquare exponential synchronization of markovian switching stochastic complex networks with time-varying delays by pinning control," Abstract and Applied Analysis, vol. 2012, Article ID 298095, 2012.

[22] Y. Tang and J.-a. Fang, "Adaptive synchronization in an array of chaotic neural networks with mixed delays and jumping stochastically hybrid coupling," Communications in Nonlinear Science and Numerical Simulation, vol. 14, no. 9-10, pp. 3615$3628,2009$.

[23] W. Wu, W. Zhou, and T. Chen, "Cluster synchronization of linearly coupled complex networks under pinning control," IEEE Transactions on Circuits and Systems. I. Regular Papers, vol. 56, no. 4, pp. 829-839, 2009.

[24] J. Wang, J. Feng, C. Xu, and Y. Zhao, “Cluster synchronization of nonlinearly-coupled complex networks with nonidentical nodes and asymmetrical coupling matrix," Nonlinear Dynamics, vol. 67, no. 2, pp. 1635-1646, 2012.

[25] J. Wang, J. Feng, C. Xu, and Y. Zhao, "Exponential synchronization of stochastic perturbed complex networks with timevarying delays via periodically intermittent pinning," Communications in Nonlinear Science and Numerical Simulation, vol. 18, no. 11, pp. 3146-3157, 2013.

[26] C. Yuan and X. Mao, "Robust stability and controllability of stochastic differential delay equations with Markovian switching," Automatica, vol. 40, no. 3, pp. 343-354, 2004. 


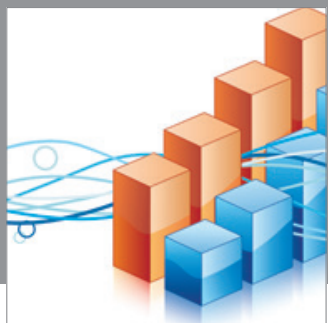

Advances in

Operations Research

mansans

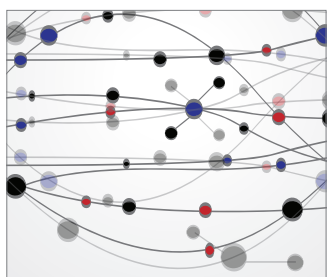

The Scientific World Journal
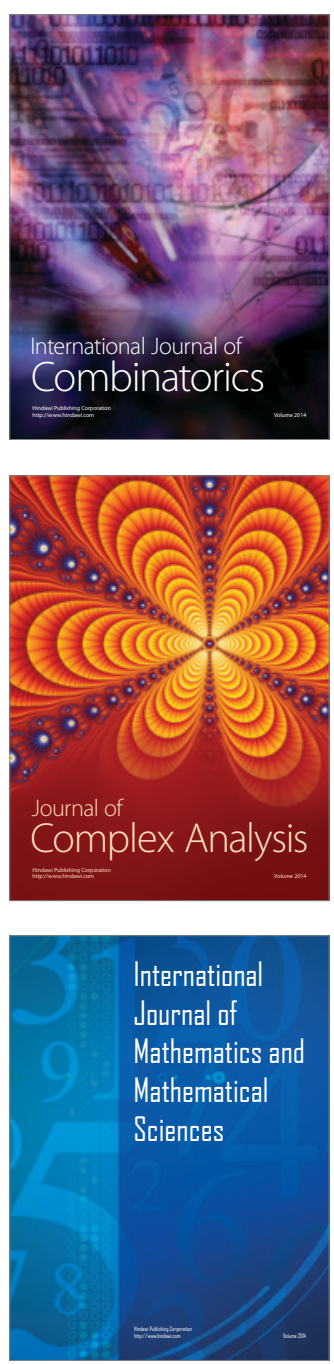
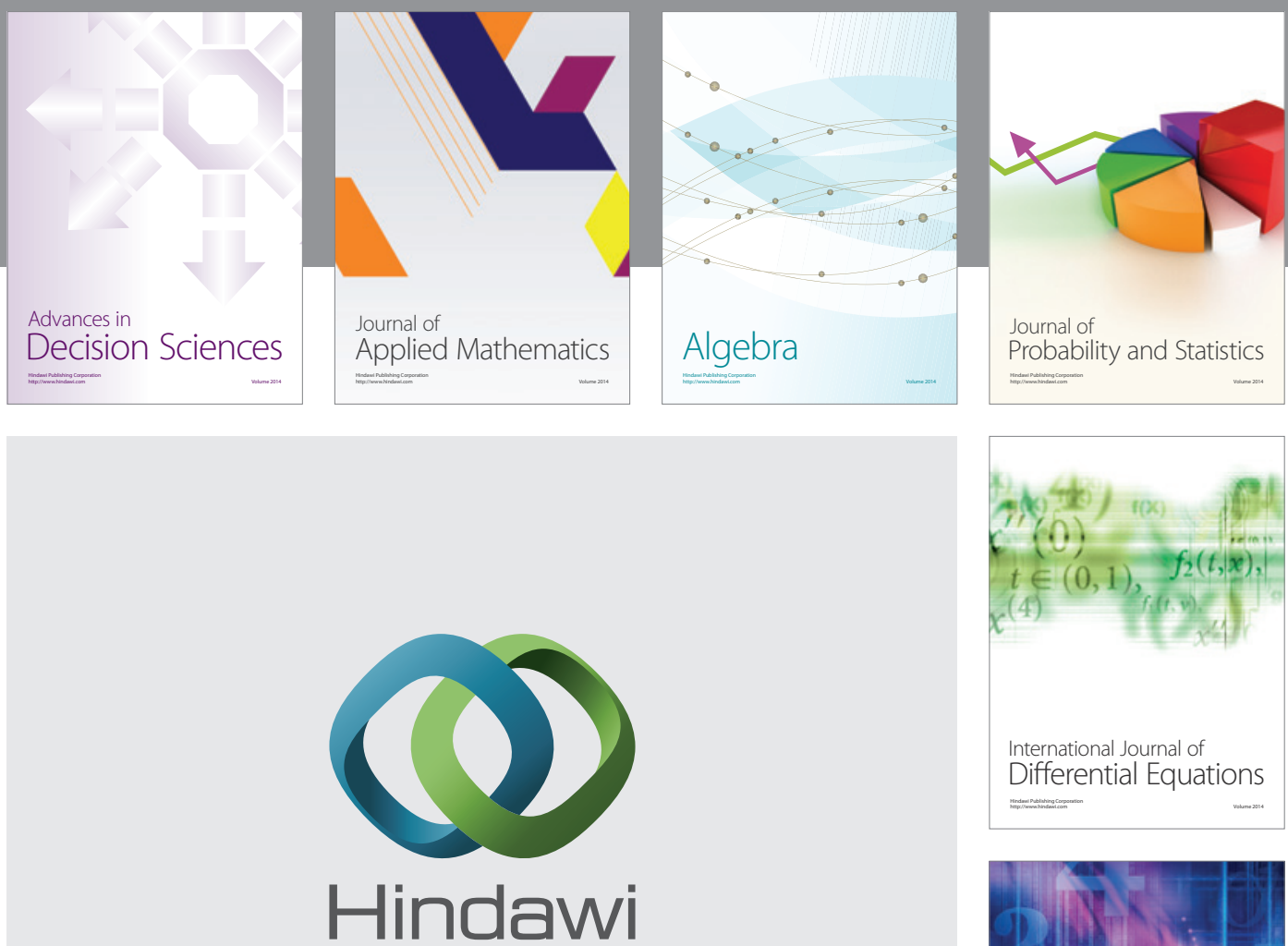

Submit your manuscripts at http://www.hindawi.com
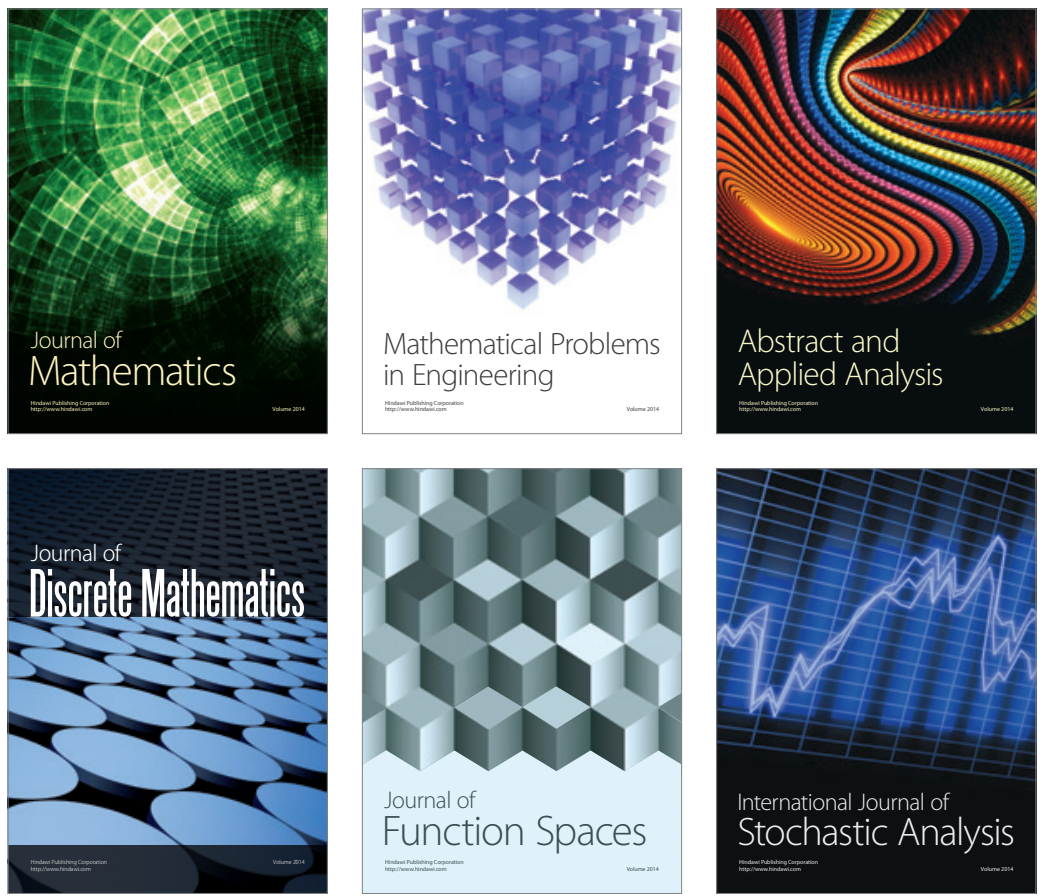

Journal of

Function Spaces

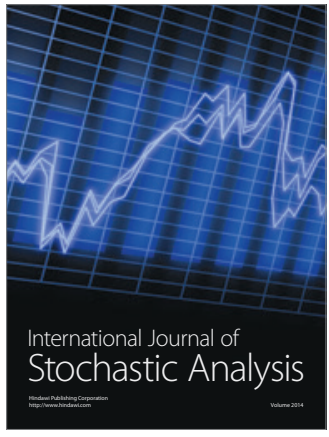

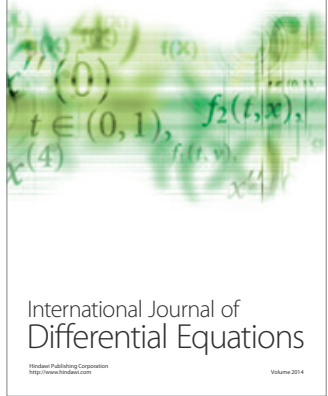
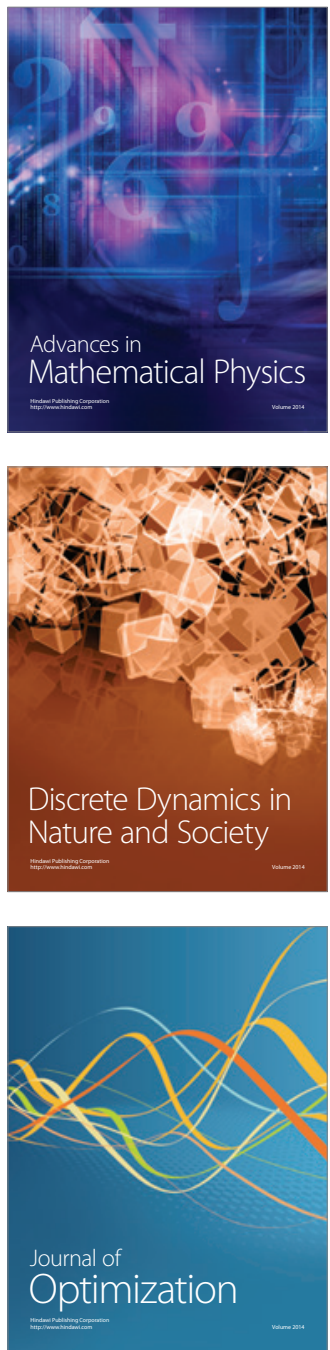